ESTUDIO

\title{
LA COMPRENSIÓN LECTORA REQUIERE CONOCIMIENTO DE VOCABULARIO Y DEL MUNDO
}

\author{
HALLAZGOS CIENTÍFICOS SOBRE EL BAJÓN \\ DE CUARTO GRADO Y EL ESTANCAMIENTO EN \\ LOS PUNTAJES NACIONALES DE COMPRENSIÓN*
}

\author{
E. D. Hirsch, Jr.
}

La comprensión lectora ha resultado ser un problema recalcitrante. Aun cuando los niños en los primeros niveles escolares pueden arreglárselas para entender textos simples, muchos estudiantes, en especial

E. D. HiRsch es fundador de Core Knowledge Foundation y profesor emérito de educación y humanidades de la Universidad de Virginia. Entre sus libros cabe destacar el ovacionado Cultural Literacy: What Every American Needs to Know y The Schools We Need and Why We Don't Have Them. Fue editor de la serie de siete volúmenes Core Knowledge Series, que abarca desde What Your Kindergartner Needs to Know hasta What Your $6^{\text {th }}$ Grader Needs to Know. La meta de la fundación Core Knowledge es la educación de excelencia e igualitaria basada en un núcleo común de aprendizaje. La fundación desarrolla programas, realiza talleres para profesores y es el eje de la creciente red de escuelas Core Knowledge.

* "Reading Comprehesion Requires Knowledge —of Words and the World: Scientific Insights into the Fourt-Grade Slump and the Nation's Stagnant Comprehension Scores” apareció en American Educator, Spring 2003, revista trimestral de la American Federation of Teachers, AFL.CIO. Su reproducción en esta edición cuenta con la autorización de American Educator y del autor. Traducido para Estudios Públicos por María José Morandé.

Véanse también en esta edición los trabajos "Las Palabras Se Aprenden Gradualmente mediante Exposiciones Múltiples”, de Steven A. Stahl y "Los Efectos de la Lectura en la Mente”, de Anne E. Cunningham y Keith E. Stanovich.

Otros artículos de E. D. Hirsch publicados en Estudios Públicos son: "La Venganza de la Realidad: Educación y las Principales Corrientes de Investigación Pedagógica”, "Crítica de una Cosmovisión”, "Los Colegios que Necesitamos y Por Qué No los Tenemos” y “Equidad y Excelencia: Metas Alcanzables en Educación”.

Estudios Públicos, 108 (primavera 2007). 
aquellos provenientes de familias de bajos ingresos, tienen serias dificultades cuando en cuarto grado deben abordar textos de estudio más avanzados. El destacado investigador E. D. Hirsch, tras haber examinado desde distintos ángulos este problema por más de tres décadas, describe en estas páginas tres hallazgos fundamentales respecto a la compresión de textos y sus alcances inmediatos para el trabajo en aula de los profesores. El primero de ellos es la necesidad de aprender a leer con fluidez. Aquí se discuten con cierto detalle los principios implícitos de la relación entre la fluidez y la compresión de textos. El segundo hallazgo es el crucial papel que juega la amplitud de vocabulario en la comprensión lectora. En este acápite se describen aspectos de la adquisición de vocabulario que no son nada obvios como podría parecer a primera vista. El tercero es la importancia del conocimiento del tema para entender lo que se lee. El autor ilustra con distintos ejemplos cuán indispensable es contar con cierto nivel de conocimiento de la materia para poder usar el vocabulario de manera eficaz.

En suma, se concluye, la comprensión no mejorará a menos que se preste especial atención a cómo se construye el conocimiento de palabras y el conocimiento del mundo. Para lograrlo, se propone revisar los programas de lenguaje y se entregan algunas pautas de cómo deberían emplearse las horas destinadas a esta asignatura. Hirsch recuerda finalmente las conclusiones del célebre sociólogo James S. Coleman luego de una vida profesional dedicada a estudiar las características de los colegios y programas efectivos. Un programa deficiente afecta más adversamente a los alumnos provenientes de familias de bajos ingresos que a los procedentes hogares de medianos ingresos, ya que estos últimos dependen menos del colegio para adquirir conocimientos. Pero un buen programa escolar es inherentemente igualitario debido al efecto compensador que tiene, precisamente, en los alumnos de hogares de bajos ingresos.

\section{M} ientras los educadores han hecho positivos avances en la enseñanza de la decodificación (esto es, convertir letras en sonidos del habla), es descorazonador que todavía no hayamos superado el "bajón de cuarto grado” de los niños en comprensión lectora. Estamos descubriendo que aun cuando la gran mayoría de los alumnos pueden arreglárselas en los primeros niveles con textos simples, muchos estudiantes, particularmente aquellos pertenecientes a familias de bajos ingresos, tienen dificultades cuando en cuarto grado deben abordar textos de estudio más avanzados. 
Para ayudar a estos alumnos debemos comprender bien dónde se origina este "bajón de cuarto grado”. “Bajón” (slump) fue la expresión con que la célebre investigadora en lectura Jeanne Chall describió la aparentemente repentina y brusca caída entre tercero y cuarto grado en los puntajes de lectura de los estudiantes de bajos ingresos. En su investigación, Chall encontró que en segundo y tercero grado los estudiantes de bajos ingresos tendían a obtener puntajes cercanos al promedio nacional en las pruebas de lectura y mediciones relacionadas, tales como ortografía y reconocimiento de palabras. Pero en cuarto grado, los puntajes de estos alumnos comenzaban a caer en forma sostenida, lo cual se hacía más pronunciado a medida que avanzaban a cursos superiores ${ }^{1}$. (Para un análisis más detallado del estudio de Chall, que marcó un hito, véase “The Fourth Grade Slump”, 2003). Describo esta caída brusca como aparentemente repentina, pues ahora hay evidencia de que ella también existe, sin medir, en los cursos anteriores. Por otro lado, hay una brecha de lenguaje — no sólo de lectura- entre los alumnos más adelantados y los más atrasados en tercero grado, por no mencionar en el segundo y el primero grado, e incluso antes.

Los investigadores han tenido conocimiento del bajón de cuarto grado en la comprensión lectora de niños pobres por muchas décadas, pero sólo recién, en especial en el trabajo de Betty Hart y Todd Risley, se cuenta con antecedentes sólidos sobre el desarrollo temprano del lenguaje en los niños $^{2}$. Ahora creemos que las pruebas de lectura hacen que la brecha de comprensión parezca mucho mayor en cuarto grado, puesto que las pruebas usadas en los cursos inferiores están dirigidas a evaluar destrezas tempranas de lectura (como la decodificación) y no intentan medir en todo su alcance las diferencias de vocabulario entre los grupos.

Sin perjuicio de lo anterior, sería un error asumir que los problemas con la comprensión se limitan a los alumnos más atrasados. De acuerdo a la evidencia más reciente del National Assessment of Educational Progress (NAEP) [Evaluación Nacional de Progreso Educativo], los puntajes en comprensión lectora de la mayor parte de los estudiantes siguen siendo bajos pese a muchos años de esfuerzos para mejorar la instrucción en lectura ${ }^{3}$. Enseñar de modo efectivo a todos los alumnos a comprender los textos escritos ha resultado ser un problema recalcitrante. Ahora que disponemos

\footnotetext{
${ }^{1}$ Chall, J. S., V. A., Jacobs \& L. E. Baldwin: The Reading Crisis. Why Poor Children Fall Behind (1990).

${ }^{2}$ Hart, B. \& T. R. Risley: Meaningful Differences in the Everyday Experience of Young American Children (1995).

${ }^{3}$ Campbell, J. R., C. M. Hombo \& J. Mazzeo: NAEP 1999 Trends in Academic Progress: Three Decades of Student Performance (2000).
} 
de buenos programas para enseñar a los niños a decodificar textos de manera precisa y fluida, la creación de programas y métodos que enseñen a los alumnos a comprender el texto con precisión y fluidez es la nueva frontera de la investigación en lectura.

Es un problema desafiante. El Departamento de Educación de los EE.UU. ha llamado a presentar propuestas de investigación para ayudar a solucionarlo. Ésa es una muy buena señal. Una renovada atención científica hacia este problema fundamental contiene la promesa de que lograremos algún día avances reales en equidad y desempeño académico. Mientras tanto, ya sabemos algunas cosas con alcances inmediatos para los profesores respecto de la comprensión lectora. Intentaré resumir algunos de los hallazgos más importantes y sus implicancias para la práctica en el aula.

\section{UN CONSENSO CIENTÍFICO CRECIENTE}

He dedicado la mayor parte de mi vida académica (desde la publicación de mi primer trabajo técnico sobre el tema en 1960) a investigar la naturaleza de la comprensión de textos: ¿Cómo sabemos que hemos comprendido correctamente un texto? ¿Acaso la lectura es una versión desplazada de la comunicación oral ordinaria? Mi vivo interés por relacionar este tema con el desempeño académico y la equidad en educación data de los 70, cuando comencé a estudiar algunos de los avances que se estaban realizando en la ciencia cognitiva y la psicolingüística (la ciencia que estudia cómo la mente produce y comprende el lenguaje hablado y escrito). Han transcurrido varias décadas en que este difícil tema de la comprensión lectora se ha venido investigando desde diversos ángulos en las humanidades y las ciencias. Ahora puedo informar que, aunque lo que todavía desconocemos excede en mucho lo que sabemos, actualmente hay consenso científico en al menos tres principios que tienen implicancias útiles para mejorar la comprensión lectora de los estudiantes. Los tres principios (que incluyen otros más) son los siguientes:

1. la fluidez permite a la mente concentrarse en la comprensión;

2. la amplitud de vocabulario aumenta la comprensión y facilita un mayor aprendizaje, $\mathrm{y}$

3. el conocimiento del tema aumenta la fluidez, amplía el vocabulario y permite una comprensión más profunda. Este principio ha sido comprendido sólo recientemente. 


\subsection{La fluidez es importante}

"Fluidez" significa "fluir”, y en este contexto también significa "rápido" o "veloz". Hay una correlación general, aunque no perfecta, entre cuán rápido se comprende un texto y qué tan bien se lo comprende. Para la mayoría de los psicólogos, incluidos aquellos que no son especialistas en lectura, sería sorprendente si no fuese así. Una persona que lee rápido ha "automatizado" muchos de los procesos subyacentes envueltos en la lectura, y puede, por consiguiente, dedicar atención consciente al significado textual más que a los procesos mismos. Es más, la fluidez se ve fuertemente favorecida por el conocimiento de palabras y el conocimiento del tema: mientras el conocimiento de palabras acelera el reconocimiento de palabras $\mathrm{y}$, por ende, el proceso de la lectura, el conocimiento del mundo acelera la comprensión del significado textual ofreciendo una base desde donde hacer inferencias ${ }^{4}$. Algunos de los principios implícitos de la relación entre fluidez y comprensión se explican a continuación.

Si la decodificación no se produce rápidamente, el material decodificado se olvidará antes de ser comprendido. ¿Han intentado ustedes comprender lo que se está diciendo en una película hablada en una lengua extranjera (por ejemplo, en francés) que han estudiado en el colegio? Aun cuando conocen las palabras, ¿acaso no es frustrante que hablen tan rápido? Mientras ustedes están tratando de entender lo que los actores acaban de decir, ellos ya están diciendo otra cosa, y la mente se satura. Respecto a la velocidad y comprensión lectora, la dificultad fundamental es todavía más seria que eso. Si fuéramos capaces de desacelerar la película para concentrarnos en identificar y traducir las palabras, encontraremos que también en esa situación nuestra comprensión sigue siendo menos que adecuada. Al tener que concentrarnos en los sonidos, transformarlos en palabras francesas y luego en palabras del castellano, tendemos a perder la trayectoria de las conexiones entre una oración y otra, y entre grupos de oraciones. Nos encontramos en la misma situación que un niño que tiene que traducir despacio y conscientemente letras a sonidos del habla. Las cosas desaparecen de nuestra mente antes de tener la oportunidad de reflexionar sobre el significado de lo que se está diciendo. Al traducir lentamente del francés al castellano nos topamos con el obstáculo de los estrictos límites de la llamada (por los cientistas cognitivos) "memoria inmediata" o "memoria de corto plazo".

${ }^{4}$ Recht, D. R. \& L. Leslie: "Effect of Prior Knowledge on Good and Poor Readers’ Memory of Text” (1988), pp. 16-20. 
Recuerdo vívidamente cuando supe por primera vez de los rigurosos límites de la memoria de corto plazo y de su importancia para la comunicación. Fue en un libro maravilloso llamado The Psychology of Communication, del distinguido cientista cognitivo George A. Miller ${ }^{5}$. El segundo capítulo ha sido uno de los artículos más célebres en el campo de la psicología, "The Magical Number Seven, Plus or Minus Two: Some Limits on Our Capacity for Processing Information” ["El mágico número siete, más dos o menos dos: Algunos límites de nuestra capacidad para procesar información"]. El "mágico número siete" resultó ser el número aproximado de ítemes (ya sean simples hechos, números o palabras que representan conceptos complejos) que se pueden retener de una vez en la mente consciente antes de que comiencen a evaporarse y terminen por olvidarse. Este "mágico número siete" es una limitación que (con algunas variantes) aflige a todas las personas, incluidos los genios. Una forma en que superamos esta limitación de la memoria de corto plazo mientras leemos es aprendiendo a activar automática y raudamente los procesos subyacentes de la lectura, de tal manera que se vuelvan rápidos e inconscientes, dejando libre a la mente consciente (esto es, la memoria de corto plazo) para pensar en el significado del texto.

Por esa razón es importante decodificar en forma rápida y certera. Los experimentos muestran que un niño que puede vocalizar el sonido de palabras sin sentido en forma rápida y exacta domina el proceso de decodificación y está en vías de liberar su memoria de corto plazo para concentrarse en la comprensión del significado. La fluidez en la decodificación se logra mediante una instrucción inicial precisa seguida de mucha práctica. Típicamente, la decodificación toma varios años de práctica antes de que los niños puedan procesar un texto impreso tan rápido como si lo estuvieran escuchando.

Los estudiantes también superan las limitaciones de la memoria de corto plazo captando rápidamente qué tipo de texto es, identificando rápidamente las palabras y entendiendo las conexiones gramaticales entre ellas y el nivel básico de la oración 6 . Este tipo de fluidez a nivel de la oración básica aumenta con la práctica y con el conocimiento de los distintos tipos de textos. Dicha fluidez general en el lenguaje también está íntimamente conectada con un conocimiento de vocabulario bien ejercitado, lo cual se refiere a cuán familiares son para el estudiante las palabras y sus distintas connotaciones. Veamos, por ejemplo, la siguiente oración: “Aparte de haber

\footnotetext{
${ }^{5}$ Miller, G. A.: The Psychology of Communication: Seven Essays (1969).

${ }^{6}$ Kintsch, W.: Comprehension: A Paradigm for Cognition (1998).
} 
pasado un largo y provechoso tiempo en las trincheras, Claire será una buena subdirectora puesto que también es capaz de mantener la vista centrada en la pelota”. Los educadores, con su conocimiento de las convenciones del lenguaje y uso del vocabulario, no tendrán problema en suponer que Claire ha trabajado con estudiantes (probablemente como profesora de colegio) y es buena para mantenerse enfocada. Pero observen que para procesar esta oración simple ustedes tuvieron que interpretar dos metáforas (trincheras y pelota); y si tuvieran que emitir un juicio de las calificaciones de Claire para subdirectora, tendrían que inferir de su conocimiento del tema las exigencias de ese trabajo específico.

Por último, la fluidez aumenta con el conocimiento del tema, permitiendo que el lector haga conexiones rápidas entre contenidos nuevos y contenidos previamente adquiridos; esto facilita tanto como profundiza la comprensión. Un experto en un tema puede leer un texto sobre ese tema en forma mucho más fluida que si lee un texto de una materia desconocida ${ }^{7}$. El conocimiento anterior de un tema acelera la comprensión básica y libera espacio en la memoria de corto plazo para hacer conexiones entre el material nuevo y la información previamente adquirida, así como para hacer deducciones y ponderar las implicaciones. Una gran diferencia entre un lector experto y uno novato - en cualquier disciplina - estriba en la capacidad del primero para incorporar aspectos básicos muy rápidamente, dejando así la mente libre para concentrarse en características importantes.

Esto se hizo palmario en un famoso experimento. El psicólogo holandés Adrian de Groot ${ }^{8}$ observó que los grandes maestros del ajedrez tienen una destreza sobresaliente que nosotros los aficionados no podemos emular. Pueden echar un vistazo de cinco segundos a una partida de ajedrez de 25 piezas en medio juego, retirarse a continuación para hacer alguna otra cosa, y luego volver para reconstruir por completo en un tablero de ajedrez en blanco las posiciones del tablero anterior sin cometer ningún error. El desempeño en esta tarea se correlaciona casi perfectamente con el ranking de los jugadores en el ajedrez. Los grandes maestros no se equivocan, los maestros muy poco, y los aficionados pueden acertar en sólo cinco o seis piezas. (¡Recuerde el mágico número siete, más dos o menos dos!) De Groot tuvo una brillante ocurrencia e hizo el mismo experimento con 25 piezas de ajedrez en posiciones que, en lugar de tomarse de un juego de ajedrez real, simplemente se colocaron al azar en el tablero. En estas nuevas circuns-

${ }^{7}$ Ericsson, K. A. \& N. Charness: "Expert Performance: Its Structure and Acquisition” (1994), pp. 725-747.

${ }^{8}$ De Groot, A.: Thought and Choice in Chess (1965). 
tancias, el desempeño de los tres grupos distintos — grandes maestros, maestros y novicios - fue exactamente el mismo, pues cada grupo recordó solamente cinco o seis piezas correctamente.

El experimento sugiere la diferencia en destreza que existe entre un lector experimentado, quien puede reproducir fácilmente las 24 letras de "el gato está sobre el felpudo", y un lector novato a quien le resulta difícil reproducir las mismas letras: e-l-g-a-t-o-e-s-t-á-s-o-b-r-e-e-l-f-e-l-p-u-d-o. Si en vez de proporcionar esa oración escrita al niño y al experto, cambiamos la tarea y les pedimos que reproduzcan una secuencia de 24 letras al azar, el desempeño del alumno de primero básico y del experto será muy semejante. En promedio, ninguno de ellos lograría construir correctamente más que una pequeña secuencia de las letras al azar. Los lectores con práctica, los grandes maestros del ajedrez y otros expertos no poseen centros cerebrales especiales que los novicios no tengan, y su desempeño no es mejor que el de los novatos en tareas estructuralmente similares y desconocidas para ambos. Sin embargo, los expertos pueden realizar proezas notables de comprensión y memoria en situaciones de la vida real, tales como recordar las posiciones en medio juego de una partida de ajedrez o el significado e incluso ortografía de oraciones y párrafos reales. ¿Cómo lo logran?

En parte lo hacen "agregando" (chunking), término empleado por George A. Miller para referirse a la manera en que las personas entendidas reúnen múltiples componentes en un solo ítem, el que a su vez se aloja en un surco independiente de la memoria inmediata. "El gato está sobre el felpudo" es una oración que se puede recordar fácilmente, y los lectores expertos pueden reproducir fácilmente las 24 letras, no porque las letras se recuerden individualmente, sino porque la oración se recuerda como un trozo (chunk) cuyos sub-elementos pueden reconstruirse a partir de un conocimiento previo del idioma escrito. Recuerden que la memoria de corto plazo puede guardar apenas siete ítemes, pero ellos pueden ser cualquier cosa, desde simples números hasta conceptos complejos previamente aprendidos que pueden concentrarse en una sola palabra o imagen. Lo que De Groot descubrió y las investigaciones posteriores han confirmado una y otra vez es que la diferencia en fluidez y destreza de orden superior entre un aficionado y un experto no radica en los músculos mentales sino en lo que De Groot denomina “erudición”, un gran acopio de conocimientos previamente adquiridos que están rápidamente disponibles, lo cual permite a la mente abarcar mucho en poco tiempo. De esta manera, cuando a los grandes maestros de ajedrez se les mostró una partida en el medio juego, ellos no estaban recordando la ubicación de las 25 piezas por separado, sino que fueron capaces de recurrir a conocimientos previos de juegos pasados simi- 
lares y a la(s) manera(s) en que las piezas estaban alineadas en dichos juegos.

Los experimentos han demostrado que cuando alguien comprende un texto, típicamente se integran sus conocimientos generales* con los significados literales de las palabras del texto para construir un modelo coherente de toda la situación implícita en el texto. Un experto puede rápidamente hacer conexiones a partir de las palabras para construir un modelo de la situación. Pero un novato dispondrá de menos conocimientos pertinentes bien estructurados, y por ende a él le tomará más tiempo construir un modelo de la situación. Supongamos que el texto contiene la expresión "Segunda Guerra Mundial”. Alguien que tiene los conocimientos necesarios sobre esa guerra podrá asimilar la expresión muy rápido y, al igual que los grandes maestros de ajedrez, será capaz de separar sus múltiples niveles o capas de significado cuando sea necesario. Los limitados conocimientos generales del novicio no estarán prontamente disponibles como los del experto y, por consiguiente, el principiante sólo hará lentamente las pocas conexiones que sus limitados conocimientos le permiten. Inevitablemente, su comprensión del texto será pobre.

\subsection{La amplitud del vocabulario es importante}

El conocimiento de vocabulario se correlaciona fuertemente con la comprensión lectora (y oral). Esto parece tan obvio que parecería inútil considerar el vocabulario en una breve reseña de las investigaciones sobre comprensión lectora. Esto es cierto. Pero sabemos algunas cosas significativas sobre la adquisición de vocabulario que podrían servir para que los alumnos aumenten su destreza en la comprensión de textos. Estas cosas no son obvias, y algunos aspectos de la adquisición de vocabulario son muy sorprendentes. A continuación se examinan algunos importantes:

En la adquisición de vocabulario, una pequeña ventaja inicial se convierte en una mucho mayor a menos que intervengamos inteligentemente para ayudar a los estudiantes más atrasados a aprender palabras a un ritmo acelerado. Hart y Risley ${ }^{9}$ han mostrado que los hogares de escasos recursos exponen a los niños menores a mucho menos palabras y a estructuras de oración mucho más simples que los hogares de clase media (véase

* Background knowledge, en el original en inglés, se ha traducido aquí y más adelante como "conocimientos generales". (N. del E.)

${ }^{9}$ Hart, B. \& T. R. Risley: Meaningful Differences in the Everyday Experience of Young American Children (1995). 
el trabajo de Hart y Risley, “The Early Catastrophe”, 2003). Un alumno de primer grado con alto rendimiento conoce cerca del doble de palabras que un alumno de bajo rendimiento y, a medida que estos estudiantes avanzan de curso, el diferencial aumenta ${ }^{10}$. En el último año de la enseñanza secundaria, los alumnos de alto rendimiento saben cerca de cuatro veces más palabras que los estudiantes de bajo rendimiento ${ }^{11}$.

La razón de esta brecha creciente está clara: los expertos en vocabulario coinciden en que una comprensión lectora adecuada depende de que una persona ya conozca cerca del 90 a 95 por ciento de las palabras de un texto ${ }^{12}$. Conocer ese porcentaje de palabras le permite al lector captar la idea principal de lo que se está diciendo y, por consiguiente, adivinar correctamente lo que las palabras desconocidas probablemente significan. Por medio de este proceso de deducción, por cierto, adquirimos el lenguaje oral en la temprana infancia y es el que sostiene el crecimiento de nuestro vocabulario en nuestras vidas.)

Lo anterior quiere decir que los mensajes que leen o escuchan los alumnos abarcan saberes y posibilidades de adquisición de palabras muy diferentes para los estudiantes más adelantados que para los más atrasados. Aquellos que saben el 90 por ciento de las palabras de un texto comprenderán su significado, y porque lo entienden también comenzarán a aprender el 10 por ciento restante de las palabras. Aquellos que no saben el 90 por ciento de las palabras, y por ende no comprenden el texto, ahora estarán mucho más rezagados en ambos frentes: perderán la oportunidad de entender el contenido del texto y de aprender más palabras. El destacado investigador en lectura Keith Stanovich se refirió a esta brecha creciente como el "efecto Mateo", del siguiente pasaje del Evangelio según San Mateo: "Porque al que tiene se le dará y tendrá en abundancia; pero al que no tiene incluso lo que tiene se le quitará".

Superar esta desventaja inicial constituye un enorme desafío. Para lograrlo, debemos recurrir a formas mejores y más eficaces de desarrollar el vocabulario. Como veremos, eso significa instruir en vocabulario de manera explícita y lo mejor posible, y proveer un ambiente que acelere la adquisi-

${ }^{10}$ Graves, M. F., G. J. Brunetti \& W. H. Slater: "The Reading Vocabularies of Primary-Grade Children of Varying Geographic and Social Backgrounds” (1982), pp. 99-104. Véase también Graves, M. F. \& W. H. Slater: "The Development of Reading Vocabularies in Rural Disadvantaged Students, Inner-City Disadvantaged and MiddleClass Suburban Students" (1987).

${ }^{11}$ Smith, M. K.: "Measurement of the Size of General English Vocabulary Through the Elementary Grades and High School” (1941), pp. 311-345.

${ }^{12}$ Nagy, W. E. y J. Scott: “Vocabulary Processes” (2000). 
ción incidental de vocabulario, que es como ocurre en su mayor parte el crecimiento del vocabulario.

Un alumno del último año de la enseñanza secundaria con buena educación conoce una vasta cantidad de palabras, en su mayoría aprendidas incidentalmente. Pero también hay un lugar importante para el desarrollo de vocabulario explícito, especialmente en los primeros años, y en particular para los alumnos que están atrasados. Isabel Beck y sus colegas ${ }^{13}$, en su excelente guía de instrucción de vocabulario explícito, estiman que se puede enseñar explícitamente unas 400 palabras por año a los alumnos en el colegio (véase “Taking Delight in Words”, 2003, p. 36, donde aparece un ejemplo de dicha instrucción). Estas 400 palabras pueden ser de enorme importancia para aquellos niños que están atrasados y que necesitan que se los nivele al punto de lograr comprender palabras clave lo más rápido posible. Pero ése es sólo el comienzo. Si queremos que todos nuestros niños comprendan bien, deben aprender muchísimas más palabras cada año por medios incidentales. Un alumno del último año de la educación secundaria que obtiene en la parte verbal del examen de aptitud SAT un puntaje suficientemente alto como para ingresar a una universidad selecta conoce entre 60.000 y 100.000 palabras. No hay coincidencia entre los expertos con respecto al número real, así que si se promedian, el número resultante sería unas 80.000 palabras. Si asumimos que un niño comienza a adquirir vocabulario a los dos años, y que un joven del último año tiene 17 años, él habrá adquirido 80.000 palabras en 15 años. Al multiplicar 365 días por 15 obtenemos 5.475 días. Si dividimos 80.000 por ese número, descubrimos que un alumno con alto rendimiento en el último curso ha aprendido unas 15 palabras por día, esto es, sobre 5.000 palabras anualmente. Pero, por supuesto, la estimación de 15 palabras por día es sólo un promedio matemático que describe un proceso poco sistemático y complejo que se da a lo largo de un frente muy amplio. (Para una breve descripción de este proceso, véase Stahl, "Las Palabras Se Aprenden de Manera Gradual mediante Exposiciones Múltiples” en esta misma edición).

La mayor parte del crecimiento del vocabulario ocurre incidentalmente, a partir de una inmersión masiva en el mundo del lenguaje y de los conocimientos. Trabajos recientes en ciencia cognitiva nos dan esperanzas de poder avanzar en este frente del aprendizaje incidental. Se ha sabido por largo tiempo que el crecimiento en el conocimiento de palabras es lento y gradual y que requiere múltiples exposiciones a las palabras. No basta apren-

${ }^{13}$ Beck, I. L., M. G. McKeown \& L. Kucan: Bringing Words to Life (2002). 
der el significado de una palabra para "adquirir" la palabra. Las denotaciones y connotaciones de las palabras así como sus modos de uso se aprenden gradualmente, poco a poco, a través de muchas, muchas experiencias de lenguaje ${ }^{14}$. El alumno del último año de la enseñanza media con alto rendimiento que sabe 80.000 palabras, las conoce con muy distintos grados de complejidad y precisión, y las ha aprendido no de a 15 por día, sino acumulando diminutos pedacitos de conocimiento de cada una de los miles de palabras con que se topa a diario. Tal como analizaré más adelante, ésta y otras consideraciones significan que debiéramos sumir a los estudiantes, por períodos prolongados, en los tipos de experiencias coherentes de lenguaje que son más conducentes a un aprendizaje eficiente de vocabulario.

\subsection{El conocimiento del tema es importante}

Para comprender la mayoría de los textos se requiere más que conocer vocabulario. Para usar eficazmente el vocabulario, el lector también necesita tener un nivel umbral de conocimiento de la materia que se está abordando; es lo que llamamos "conocimiento del tema". Consideremos los siguientes ejemplos.

El conocimiento del tema permite a los lectores dar sentido a las combinaciones de palabras y escoger entre los múltiples posibles significados de una palabra. Un típico artículo de periódico demuestra por qué es importante saber anticipadamente algo acerca del tema de un texto para comprenderlo. Si estamos leyendo un relato sobre un juego de béisbol en la sección deportes de un periódico, típicamente debemos saber bastante sobre el béisbol para comprender lo que se está diciendo. Piensen en la cantidad de conocimientos sobre el béisbol que ya debe tenerse en mente para entender la siguiente oración simple: "Jones se sacrificó y golpeó en una corrida”. Unidas así, las palabras literales casi no tienen sentido. Alguien que no sabe nada acerca del béisbol y que lee esta oración estaría confundido, aun cuando no hubiera ningún problema con su fluidez o conocimiento general de palabras como "sacrificó". Las palabras tienen múltiples intencionalidades y significados, y su significado en un caso particular depende del conocimiento del tema que tenga el lector. La palabra "sacrificio" tiene distintas connotaciones en un relato de béisbol y en la Biblia.

El conocimiento del tema es necesario para dar sentido a oraciones que de otra forma serían confusas. Una vez leí una anécdota acerca de una

${ }^{14}$ Nagy, W. E. y J. Scott: “Vocabulary Processes” (2000). 
persona mayor que asistió a una conferencia sobre la relatividad pronunciada por el gran Albert Einstein en la Universidad de Princeton: "Comprendí todas las palabras. Sólo que la manera en que aparecían unidas me desconcertó”. Lo que esta persona daba a entender es que las palabras cotidianas que Einstein empleó en su conferencia se referían a un conocimiento de una materia específica. Si no conocemos dicha materia, no podemos construir un modelo mental que tenga sentido respecto de lo que se está diciendo. La siguiente es una oración de Einstein como la que se podría haber oído en la conferencia: "Se verá a partir de estas reflexiones que al seguir la teoría general de la relatividad llegaremos a una teoría de la gravedad, puesto que somos capaces de producir un campo gravitacional meramente cambiando el sistema de coordenadas". Conozco todas estas palabras, pero debido a que no puedo imaginar cómo el hecho de cambiar las coordenadas "producirá" gravedad, no puedo entender lo que significa esa oración.

En un plano más cotidiano, tomemos esta oración del número de febrero de 2003 del National Geographic: "Gigante y luminosa, la primera estrella se formó como una perla en el interior de capas de gas en torbellino". La mayor parte de los adultos puede comprender esta oración recurriendo a su conocimiento de la teoría del big bang, la formación de las perlas (como también del uso de metáforas, sobre lo cual volveré más adelante) y los gases. Pero esperaríamos diferentes grados de comprensión entre, por ejemplo, físicos, astrónomos aficionados, y ustedes y yo. Igualmente, cabría esperar escasa comprensión por parte de los alumnos promedio de sexto grado, no sólo por las palabras utilizadas sino porque en este contexto esas palabras representan un vasto conocimiento del tema.

Cuando alguien lee (y escucha) debe hacer deducciones que dependen de conocimientos previamente adquiridos, y no de habilidades "deductivas” contextualizadas. Muchos textos básicos de lectura instan a los profesores a que dediquen valiosas horas pedagógicas a instruir a los estudiantes para que desarrollen "habilidades deductivas". Pero un ejemplo simple ilustra que la deducción en sí misma es una habilidad bastante básica que los niños ya poseen. Si alguien le dice a un niño: “iOye, cállate! Estoy tratando de leer”, la mayoría de los niños, ya sean más adelantados o más atrasados, pueden deducir la conexión entre la primera oración y la segunda. Saben de antemano que el hecho de escuchar a alguien hablar puede distraer y dificultar la lectura. Así que pueden construir un modelo mental que, con sentido, conecta la oración “¡Oye, cállate!” con la oración "Estoy tratando de leer". Pero muchos niños pueden no entender la siguiente oración simple: "Quise ir de vacaciones a México este año, pero mi señora sólo 
puede tomarse vacaciones en julio". A los niños que no pueden entender la conexión entre las cláusulas no les falta habilidad deductiva; simplemente no tienen el conocimiento geográfico de que México es en extremo caluroso en julio y, por ende, no es el mejor destino para unas vacaciones placenteras.

Al hablar y escribir siempre se entregan significados que las palabras en sí mismas no comunican explícitamente. Si quienes hablan o escriben tratasen de hacer que todo fuese explícito, tardarían mucho en decir cualquier cosa, y el mensaje se volvería larguísimo y se apartaría del tema principal. Desde la infancia aprendemos que la comprensión del lenguaje oral obliga a los lectores a construir activamente significados proveyendo los conocimientos faltantes y haciendo deducciones. Por supuesto, la necesidad de conocimientos previos no es exclusiva de la comunicación oral; también es necesaria para comprender textos escritos.

Desde la década de 1960 varios investigadores han venido señalando que para comprender se requiere hacer deducciones mediante la activación del conocimiento ya existente del tema. Pero la noción básica se remonta a mucho antes. En la antigüedad griega se entendía que la comunicación involucra hacer deducciones basadas en conocimientos que se dan por sentado. El término griego para dicho argumento implícito era enthymeme o entimema, de en (en) y thumos (mente), es decir, algo que se tiene en mente y se da por sentado, pero no se expresa ${ }^{15}$. Un ejemplo de esta característica del habla es un silogismo trunco: "Todos los hombres son mortales, así que Sócrates es mortal”. Para que esta afirmación tenga sentido lógico debemos deducir la premisa que falta, a saber, que Sócrates es un hombre.

Igualmente, la comprensión lectora también depende de que el lector llene los espacios en blanco y provea, en silencio, la suficiente cantidad de premisas no expresadas para que adquiera sentido coherente lo que se está leyendo. Una vez que el texto escrito se ha decodificado y convertido en palabras, la comprensión lectora, al igual que la comprensión oral, requiere la activa construcción de deducciones a partir de expresiones repletas de premisas no expresadas y alusiones sin explicar.

\section{Se requieren conocimientos generales (background knowledge)} para comprender las ironías, las metáforas y otras figuras literarias. Aparte de realizar conexiones lógicas, hay otros medios para activar los conocimientos generales pertinentes que son necesarios para reconstruir el signifi-

${ }^{15}$ Singer, M., R. Revlin \& M. Halldorson: "Bridging-Inferences and Enthymemes” (1990), pp. 35-52. 
cado de un texto. Uno de los ejemplos más inmediatamente obvios es la ironía, que, por definición, se abstiene de expresar su significado. Si lo hiciese, dejaría de ser ironía y se convertiría en una afirmación explícita. “Es un niño brillante.” ¿Es una afirmación directa, en cuyo caso se piensa que el niño es inteligente, o es irónica, en cuyo caso se piensa que es estúpido? La ironía está sujeta a dos interpretaciones contrarias, la directa y la irónica. Para decidir entre estas dos posibilidades, el lector tiene que activar el conocimiento pertinente del mundo que no se ha expresado en la oración.

Otra ilustración importante del modo en que los conocimientos generales se activan en el proceso de comprensión del lenguaje es la metáfora, un elemento casi omnipresente del habla. Los estudiantes entienden fácil y rápidamente "La victoria es dulce”. Lo mismo ocurre con "La guerra es el infierno” y "No seas aguafiestas”. Sabemos que su intención no es literal, puesto que sabemos a qué se refieren. Los investigadores han mostrado que las metáforas a menudo se procesan en forma tan rápida como el significado literal, lo que indica que constantemente estamos activando los conocimientos generales en la comprensión. En la segunda parte de este artículo mostraré que esta idea del conocimiento que se da por sentado es una clave importante del tipo de instrucción que puede ayudar a que los estudiantes mejoren su habilidad para comprender textos escritos.

En los últimos años, los esfuerzos para acrecentar la lectura se han enfocado en cómo enseñar mejor a decodificar. Y, desde luego, la decodificación fluida es un prerrequisito absoluto de la comprensión. Pero podemos ver a partir de este rápido resumen de investigación que la comprensión —el objetivo de la decodificación — no mejorará a menos que prestemos especial atención a la construcción del conocimiento de palabras y conocimiento.

\section{REPLANTEANDO EL PROGRAMA DE LENGUAJE}

Para mejorar la lectura, los colegios en los EE.UU. han ido aumentando constantemente el tiempo dedicado a Lenguaje. Por ejemplo, en Baltimore, Chicago y todo el estado de California los profesores de los primeros niveles deben dedicar diariamente dos horas y media a Lenguaje. En una encuesta de la AFT (American Federation of Teachers), el 80 por ciento de los profesores básicos dijo que sus colegios recomendaban un bloque de Lenguaje de dos horas o más al día. (Si la encuesta se hubiese limitado a profesores de los cursos básicos inferiores, el porcentaje podría haber sido mayor aún.) Incluso pese al gran desafío que tenemos, es una enorme canti- 
dad de tiempo, en especial si se tiene en cuenta que se trata por lo general de las preciadas horas de la mañana. Necesitamos emplear el tiempo en forma óptima. Tal como veremos, no lo estamos haciendo. ¿Qué ocurre en esas horas? Considerando lo que ya hemos analizado acerca de la comprensión lectora, ¿cómo debería emplearse?

\subsection{Comenzar tempranamente a construir el conocimiento de palabras y del mundo}

Tal como lo mencioné antes, el típico niño más atrasado ingresa al jardín infantil conociendo sólo la mitad de las palabras que sabe un típico niño más adelantado. Debido al efecto Mateo, tal vez nunca se podrá superar por entero esta enorme desventaja original. Tal como hemos visto, a partir de las mismas experiencias de lenguaje los niños con un vocabulario rico aprenden más vocabulario y contenido que los niños con un vocabulario pobre. Por otra parte, es posible remediar de manera inteligente esta situación, en especial si se comienza tempranamente estimulando un crecimiento óptimo del vocabulario desde el nivel preescolar. El conocimiento de palabras y temas se adquiere mediante un proceso gradual y acumulativo. Puesto que un aprendizaje temprano de palabras y cosas es la única manera de superar una desventaja temprana, el argumento a favor de incluir un contenido óptimo en Lenguaje parece convincente.

Enseñar tempranamente a decodificar a través de textos simples “decodificables” que permitan al niño progresar con rapidez en la habilidad de decodificación presenta poderosas ventajas teóricas y prácticas. Pero la mejor investigación en este ámbito sugiere que cuarenta minutos de instrucción diaria de decodificación es suficiente en primero básico; y para la mayor parte de los alumnos de segundo básico, veinte minutos es un tiempo adecuado $^{16}$. Esto deja entre una y dos horas diarias (dependiendo del tiempo asignado a Lenguaje) para actividades que fomenten el vocabulario, el conocimiento de temas diversos y la fluidez. Dichos conocimientos podrían transmitirse a través de lecturas en voz alta, una instrucción bien concebida de vocabulario y diversas actividades que sumerjan a los niños en el conocimiento de las palabras y del mundo. Pero ningún programa básico que se haya publicado del que yo tenga conocimiento persigue sistemáticamente este objetivo. Abundan las oportunidades perdidas.

\footnotetext{
${ }^{16}$ Comunicación personal con Louisa Moats.
} 


\subsection{Construcción de la comprensión oral y de una base de conocimientos generales (background knowledge)}

Thomas Sticht ha mostrado que la comprensión oral típicamente fija un límite superior a la comprensión lectora. Si no se reconoce y comprende una palabra cuando se la escucha, ésta tampoco se comprenderá durante la lectura ${ }^{17}$. Esto nos dice algo muy importante: la comprensión oral debe desarrollarse en general en nuestros estudiantes más jóvenes si queremos que ellos sean buenos lectores.

Desde la más temprana edad, la lectura es mucho más que decodificar. Desde el comienzo, leer es también, además de adquirir, acceder a conocimiento del lenguaje y conocimiento de temas. Lo anterior quiere decir que la instrucción y la práctica para decodificar con fluidez necesitan ir de la mano de la instrucción y práctica del conocimiento de vocabulario y de temas diversos. Si deseamos mejorar el rendimiento posterior y evitar el bajón de cuarto grado, es preciso combinar una instrucción temprana en los procedimientos de lectura y escritura con una instrucción temprana en el contenido de lo que se lee y escribe, específicamente: vocabulario, convenciones del lenguaje y conocimiento del mundo.

En los primeros cursos, antes de que los alumnos puedan leer textos sustantivos por su cuenta, el contenido se transmite mejor oralmente. Un medio importante son las lecturas en voz alta hechas por el profesor, en que los textos seleccionados, conforme a su interés, tema y vocabulario, se leen en voz alta, seguidos de discusiones y lecciones que forjan la comprensión de las ideas, asuntos y palabras del cuento. Tal como se ilustra en el trabajo de Kate Walsh, “Lost Opportunity” (2003), la mayor parte de las series más populares de lectura inicial incluyen en sus programas lecturas en voz alta, pero el contenido es casi siempre trivial, y las lecturas en voz alta generalmente se eliminan en segundo grado, aun cuando las investigaciones han demostrado que los estudiantes se benefician con las lecturas en voz alta hasta octavo grado ${ }^{18}$. Además, las guías para profesores de las series iniciales instruyen a los profesores sobre cómo construir una base de conocimientos, pero por lo general en temas enteramente banales, como las mascotas, el compartir e incluso qué alimentos untables saben mejor en las tostadas.

\footnotetext{
${ }^{17}$ Sticht, T. G.: “Auding and Reading: A Developmental Model” (1975).

${ }^{18}$ Chall, J. S. \& V. A. Jacobs: "The Reading, Writing, and Language Connection” (1996), pp. 33-48.
} 
Otro problema es que los programas de Lenguaje de los primeros grados, tanto en términos de lecturas en voz alta como de decodificación de textos, se dedican abrumadoramente a la ficción. La literatura es un conocimiento de dominio muy importante por derecho propio, pero no he visto ninguna refutación convincente al argumento de Jeanne Chall, quien escribió que necesitamos poner mucho más énfasis en la no ficción en las clases de Lenguaje de los primeros cursos. Este énfasis es fundamental para que los niños aprendan las palabras y los conceptos que les permitirán entender periódicos, revistas y libros dirigidos al público general ${ }^{19}$. Pero el problema no sólo radica en el acento desproporcionado en la ficción; además, la ficción que se les ofrece es típicamente trivial en contenido y simple en sus convenciones del lenguaje. La ficción puede contribuir a conocer y comprender pueblos, países, épocas e ideas que son muy importantes y que sin embargo los niños desconocen por completo. Un excelente ejemplo de este tipo de ficción es The Hole in the Dike (El agujero en el dique), incluida en una serie de lectura inicial. La famosa leyenda familiariza a los estudiantes con Holanda, su geografía, el poder del agua y el ingenioso sistema de diques para retenerla. Pero ficción de este tipo es la excepción. Mucho más típicos, especialmente entre tercero y cuarto grado, son los cuentos basados en el aquí y ahora que transmiten prosaicamente "ideas" que los niños ya conocen: el colegio, la amistad, la familia y cosas por el estilo.

\subsection{No dedicar demasiado tiempo a enseñar habilidades formales de comprensión}

Se está destinando demasiado tiempo de las clases de Lenguaje a enseñar a los niños estrategias formales de comprensión tales como predicción, clasificación y búsqueda de la idea principal (véase K. Walsh, "Lost Opportunity”, p. 24). En la mayor parte de los textos de Lenguaje estos ejercicios se prolongan durante todo un año y varios años más. Los investigadores creen que la práctica de estas estrategias de comprensión son beneficiosas al comienzo. Éstas les enseñan a los niños a interpretar un texto buscando su significado de la misma manera en que ellos ya interpretan el lenguaje oral de los adultos y de sus pares. Lo cual ayuda a que los niños entiendan que el texto, al igual que una persona, está tratando de comunicar algo. Pero después de un beneficio inicial, aumentar deliberadamente la práctica de estas habilidades formales es una pérdida de tiempo, según

${ }^{19}$ Chall, J. S., V. A., Jacobs \& L. E. Baldwin: The Reading Crisis. Why Poor Children Fall Behind (1990). 
concluye Barak Rosenshine a partir de las investigaciones sobre el empleo de estos métodos y sus efectos. Rosenshine descubrió que destinar seis clases para enseñar estas habilidades tenía el mismo efecto en la comprensión lectora de los alumnos que destinar 25 clases para dicho fin. Tras un rápido salto inicial los efectos positivos alcanzan una planicie o techo, más allá del cual se obtienen muy pocos beneficios adicionales ${ }^{20}$.

El hallazgo de Rosenshine podría haberse predicho a partir del resto de lo que sabemos acerca de la comprensión. Los niños han estado la mayor parte de sus vidas infiriendo estratégicamente el significado del habla. (Recuerden: todos los niños pueden interpretar la deducción implícita en “ $i$ Cállense! Estoy tratando de leer”.) A los estudiantes les hace falta mucho más conocimiento del tema pertinente que técnicas de deducción. Pero aun cuando es bueno dedicar sólo una pequeña cantidad de tiempo a enseñar explícitamente habilidades de comprensión, esto no quiere decir que estas habilidades luego serán abandonadas. Ellas se activarán a medida que haya una mayor familiaridad con el vocabulario y la materia sobre la cual se está leyendo. La finalidad de una estrategia de comprensión consiste en activar el conocimiento pertinente del estudiante de manera de construir un modelo de la situación. Esto es magnífico, pero si el conocimiento previo pertinente no está presente, las estrategias de comprensión consciente no pueden activarlo.

\subsection{Construcción sistemática del conocimiento de palabras y del mundo}

Veamos por qué los actuales programas no han podido mejorar los puntajes en comprensión lectora. En primer lugar, no han podido mejorar significativamente el vocabulario de los estudiantes. Los investigadores en vocabulario coinciden en que para tener un buen comienzo en el aprendizaje de las connotaciones de una palabra, una persona necesita múltiples exposiciones a la palabra en diferentes contextos. Esa exposición no ocurre cuando a los niños se les entrega una selección fragmentada de lectura en que los temas saltan de un día en la playa a una visita a la sección verduras del supermercado.

Ése es el defecto más superficial de los programas actuales; hay otro más profundo. Con su marcada orientación a la literatura trivial, estos programas no fomentan en forma coherente el conocimiento general de los estudiantes. Un vocabulario amplio y un vasto conocimiento van de la mano.

${ }^{20}$ Rosenshine, B. \& C. Meister: "Reciprocal Teaching: A Review of the Research” (1994), pp. 479-530. 
El lenguaje no es una esfera aislada de actividad sino nuestra herramienta humana fundamental para relacionarnos con el mundo. La mejor manera de ampliar el lenguaje de los alumnos es ampliando su comprensión de aquello a lo que el lenguaje refiere. Si deseamos que los estudiantes sepan las connotaciones de la palabra “manzana”, la mejor instrucción incluirá referencias a verdaderas manzanas —no sólo a asociaciones verbales como “dulces”, “redondas” y “firmes”, sino a los objetos reales que unifican esas características. Un programa ideal de Lenguaje es un programa de conocimientos. Es un programa que asegura y consolida el significado de las palabras en las mentes de los estudiantes en virtud de que ellos saben a qué se refieren realmente las palabras.

A la difunta Jeanne Chall le afligía el nulo conocimiento del mundo que entregan los textos de ficción esquemáticos y dispersos que poco o nada ayudan a que los alumnos amplíen sus conocimientos y vocabulario. Señalaba que el conocimiento del mundo es un componente esencial de la comprensión lectora, puesto que cada texto da por sentado la familiaridad de los lectores con una amplia gama de hechos no verbalizados ni escritos acerca del mundo cultural y natural.

Hoy en día se reconoce que la causa principal de la brecha de rendimiento entre los grupos socioeconómicos es una brecha de lenguaje. Buena parte de los trabajos dedicados al lenguaje y el vocabulario descuidan un elemento fundamental de la adquisición de las palabras que por ser tan básico resulta casi invisible: la relación entre el lenguaje y el conocimiento del mundo al que se refiere el lenguaje es extremadamente fuerte. En los seres humanos, el conocimiento de un tema va automáticamente acompañado del uso del lenguaje que representa ese conocimiento. Este nexo lenguaje-conocimiento establece el principio clave de un programa de Lenguaje: un programa coherente y extenso es el que construye vocabulario con mayor eficacia y el que más contribuye a una mejor comprensión lectora.

Lo que más sirve para el desarrollo de la comprensión lectora y el vocabulario en el aula es destinar largo tiempo a leer y escuchar textos sobre el mismo tema y a discutir los hechos e ideas contenidos en ellos. La cantidad de clases dedicadas a un tema debiera estar determinada por el tiempo necesario para entender y familiarizarse con el tema - y por el nivel de enseñanza. En preescolar y en primero básico, los alumnos podrían escuchar y comentar un tema en particular durante sólo tres clases. En cuarto grado, la inmersión podría demorar dos semanas, y en cursos superiores, más tiempo. Es innecesario decir que este principio se aplica a buenos relatos tanto de ficción como de no ficción. Estos textos y temas deben ser lo suficientemente motivadores para que profesores y alumnos deseen hablar 
acerca de lo que leyeron, y lo bastante profundos para que haya suficientes motivos para volver sobre el tema.

Semejante inmersión en un tema no sólo mejora la lectura y desarrolla el vocabulario; también desarrolla la habilidad de la escritura. Uno de los descubrimientos notables que hice durante los numerosos años que enseñé composición fue cómo mejoró la escritura de mis alumnos cuando nuestra clase siguió un tema interesante por un período largo. La organización de sus ensayos mejoró. Su ortografía mejoró. Su estilo mejoró. Sus ideas mejoraron. Ahora entiendo por qué: cuando la mente se familiariza con un tema, sus limitados recursos pueden volcarse a otros aspectos de la tarea de escribir, tal como sucede en la lectura. Todos los aspectos de una habilidad crecen y se desarrollan a medida que la familiaridad con el tema aumenta. Al enseñar una materia necesariamente se enseñan destrezas, matándose así varios pájaros de un tiro.

Asimismo, hay evidencia de que al enseñar contenidos sólidos en las clases de lectura, aumenta la comprensión lectora de los estudiantes en forma mucho más eficaz que con cualquier otro método. Un trabajo de investigación muy sugerente de John Guthrie y sus colegas muestra que una instrucción en lectura que se concentra en un ámbito coherente del conocimiento por un período prolongado favorece no sólo el vocabulario general de los estudiantes, también mejora su fluidez y motivación para leer ${ }^{21}$. Esto es exactamente lo que podríamos predecir a partir de lo que se ha determinado sobre los procesos de la comprensión lectora y el crecimiento de vocabulario. Por ejemplo, tomemos la regla general de que es necesario conocer el noventa por ciento de las palabras para comprender un texto. A medida que la exposición a un tema específico se prolonga en el tiempo, mayor será el porcentaje de palabras que un niño conocerá de un texto. Esto quiere decir que el aprendizaje incidental de todas las palabras de un texto, tanto generales como específicas, se verá continuamente favorecido mediante una inmersión prolongada en una materia. Al mismo tiempo, la fluidez general también crecerá a medida que el niño se familiarice más con el tema. En resumen, el principio de inmersión en el contenido puede hacer que las clases de Lenguaje se conviertan en experiencias más interesantes para los alumnos y también en medios más efectivos para estimular sus habilidades de lectura y escritura.

El destacado sociólogo James S. Coleman, tras una vida profesional dedicada a examinar las características de los colegios y programas efecti-

${ }^{21}$ Guthrie, J. T., E. Anderson, S. Alao \& J. Rinehart: "Influences of ConceptOriented Reading Instruction on Strategy Use and Conceptual Learning from Text” (1999), pp. 343-366. Ver también Guthrie, John T., Allan Wigfield \& Clare VonSecker: "Effects of Integrated Instruction on Motivation and Strategy Use in Reading (2000), pp. 331-341. 
vos, concluyó que el rasgo más importante de un buen programa educativo consiste en hacer buen uso académico de las horas escolares. El tema permanente del trabajo de Coleman fue la "igualdad de oportunidades educativas" - título de su monumental "Informe Coleman”, de 1966²2. Hacer buen uso de las horas escolares, concluyó, es la función individual más igualitaria que los colegios pueden llevar a cabo, dado que para los alumnos más atrasados el tiempo que pasan en el colegio es la única instancia de aprendizaje académico, en tanto que los alumnos aventajados aprenden mucho fuera del colegio. En general, la principal conclusión que se extrajo del trabajo de Coleman fue que una situación social favorable tenía más peso en el rendimiento académico que el trabajo escolar, tal como estaban conformados los colegios entonces. Pero hubo un segundo y más esperanzador hallazgo en el "Informe Coleman" al cual el mismo Coleman se abocó a continuación: el carácter inherentemente igualitario y compensatorio de un programa escolar realmente bueno. Un programa deficiente afecta adversamente a los alumnos de bajos ingresos más que a los de ingresos medianos, quienes dependen menos del colegio para adquirir conocimientos. Por el contrario, un buen programa es inherentemente compensatorio porque tiene un mayor efecto en los alumnos de bajos ingresos que en los de ingresos medianos. Esto es así dado que los alumnos de bajos ingresos tienen más que aprender y con un programa efectivo comienzan a ponerse al día.

Un buen y efectivo programa de Lenguaje enfocado a conocimientos generales y que hace uso eficaz de las horas en el colegio no sólo mejorará los logros en lectura para todos los estudiantes sino que, en virtud del principio de Coleman, reducirá la brecha en lectura —así como la brecha de rendimiento— entre los diferentes grupos.

\section{REFERENCIAS BIBLIOGRÁFICAS}

Beck, Isabel L., Margaret G. McKeown \& Linda Kucan (2002): Bringing Words to Life, New York: Guilford Press.

“Taking Delights in Words: Using Oral Language to Build Young Children’s Vocabularies”. En American Educator, Spring 2003.

Campbell, J. R., C. M. Hombo \& J. Mazzeo (2000): NAEP 1999 Trends in Academic Progress: Three Decades of Student Performance. NCES 2000469.

Chall, J. S. (2003): “The Fourth Grade Slump”. En American Educator, Spring 2003.

Chall, J. S. \& V. A. Jacobs (1996): “The Reading, Writing, and Language Connection”. En J. Shimron (ed.), Literacy and Education: Essays in Memory of Dina Feitelson, pp. 33-48. Cresskill, N. J.: Hampton Press, Inc.

${ }^{22}$ Coleman, J. S.: Equality of Educational Opportunity (1966). 
Chall, J. S., V. A., Jacobs \& L. E. Baldwin (1990): The Reading Crisis. Why Poor Children Fall Behind. Cambridge, Mass.: Harvard University Press.

Coleman, J. S. (1966): Equality of Educational Opportunity. U. S. Department of Health, Education and Welfare, Office of Education, Print Office, Washington, D. C.

De Groot, A. (1965): Thought and Choice in Chess. The Hague: Mouton and Company. [Disertación doctoral publicada en holandés en 1946.]

Ericsson, K. A. \& N. Charness (1994): "Expert Performance: Its Structure and Acquisition”. En American Psychologist, Vol. 49 (8).

Graves, M. F., G. J. Brunetti \& W. H. Slater (1982): "The Reading Vocabularies of Primary-Grade Children of Varying Geographic and Social Backgrounds”. En J. A. Jarris \& L. A. Harris (eds.), New Inquiries in Reading Research and Instruction, pp. 99-104. Rochester, N.Y.: National Reading Conference.

Graves, M. F. \& W. H. Slater (1987): “The Development of Reading Vocabularies in Rural Disadvantaged Students, Inner-City Disadvantaged and Middle-Class Suburban Students”. Documento presentado en la reunión de la American Educational Research Association, Washington, D.C.

Guthrie, John T., Allan Wigfield \& Clare VonSecker (2000): "Effects of Integrated Instruction on Motivation and Strategy Use in Reading. En Journal of Educational Psychology, Vol. 92 (2), June 2000, American Psychological Association.

Guthrie, J. T., E. Anderson, S. Alao \& J. Rinehart (1999): "Influences of ConceptOriented Reading Instruction on Strategy Use and Conceptual Learning from Text”. En Elementary School Journal, Vol. 99 (4), March.

Hart, Betty \& Todd R. Risley (1995): Meaningful Differences in the Everyday Experience of Young American Children. Baltimore, Md.: Paul H. Brookes Publishing Co.

“The Early Catastrophe”. En American Educator, Spring 2003.

Kintsch, W. (1998): Comprehension: A Paradigm for Cognition. N. Y.: Cambridge University Press.

Miller, G. A. (1969): The Psychology of Communication: Seven Essays. Baltimore, Md.: Penguin Books.

Nagy, W. E. y J. Scott (2000): “Vocabulary Processes”. En M. Kamil et al., Handbook of Reading Research, Vol. III. Mahwah, N. J.: Erlbaum.

Recht., D. R. \& L. Leslie (1988): "Effect of Prior Knowledge on Good and Poor Readers’ Memory of Text”. En Journal of Educational Psychology, Vol. 80 (1), American Psychological Association.

Rosenshine, B. \& C. Meister (1994): "Reciprocal Teaching: A Review of the Research". En Review of Educational Research, Vol. 64, Winter.

Singer, M., R. Revlin \& M. Halldorson (1990): "Bridging-Inferences and Enthymemes”. En A. C. Graesser \& G. H. Bower (eds.), Inferences and Text Comprehension, pp. 35-52. San Diego, California: Academic Press.

Smith, M. K. (1941): "Measurement of the Size of General English Vocabulary Through the Elementary Grades and High School”. En Genetic Psychological Monographs, 24. 
Stahl, Steven (2003): "How Words Are Learned Incrementally Over Multiple Exposures”. En American Educator, Spring 2003. [Versión en castellano en Estudios Públicos, 108, Centro de Estudios Públicos, www.cepchile.cl]

Sticht, T. G. (1975): “Auding and Reading: A Developmental Model”. En Catalog of Selected Documents in Psychology, Vol. 5, Winter.

Walsh, Kate (2003): “Lost Opportunity”. En American Educator, Spring 2003. 